\title{
Sistema de Avaliação de Desempenho Baseado em Controle Adaptativo: uma Aplicação Participativa
}

\section{Performance Appraisal System Based on Adaptive Control: a Participatory Application}

\author{
Alisson Antonio de Oliveira, \\ Instituto Federal do Paraná - IFPR - Brasil \\ alissonantonio@yahoo.com.br \\ ORCID: http://orcid.org/0000-0001-9961-1730 \\ Gutemberg Ribeiro \\ Instituto Federal do Paraná - IFPR - Brasil \\ gutemberg.ribeiro@ifpr.edu.br \\ https://orcid.org/0000-0002-4755-5498
}

Submetido em 05/08/2019; Aprovado em 31/10/2019.

\begin{abstract}
Resumo
Objetivo - Este trabalho tem como objetivo caracterizar a viabilidade de utilizar um sistema de avaliação de desempenho baseado em Controle Adaptativo. Metodologia / abordagem - Será demonstrando com um exemplo genérico a racionalidade do sistema. Este exemplo destaca os problemas e soluções para uma avaliação de desempenho eficaz que pode ser aplicado via plataforma WEB gerando não apenas um sistema de avaliação de desempenho, mas também de feedback orientado as pessoas em início ou fim de carreira, uma vez que ele possui a capacidade de adaptar o feedback ao nível do trabalho e a área do trabalho. Constatações - Com a construção detalhada do modelo, a viabilidade do sistema é demonstrada de maneira metódica, assim como ficou evidente a necessidade de novas pesquisas visando à popularização do sistema adaptativo. Contribuições - A avaliação de desempenho é de fundamental importância para o resultado organizacional. No entanto, observa-se que os sistemas tradicionais não conseguem medir efetivamente o desempenho, seja individual, grupal ou organizacional. 0 foco do modelo é para organizações co-participativas e de gestão pública, entretanto pode ser usado em instituições privadas também.
\end{abstract}

Palavras Chave: Sistema de avaliação de desempenho, Sistemas adaptativos, Co-participação, Administração pública, Gestão de desempenho

\begin{abstract}
Purpose - This paper aims to characterize the feasibility of using a performance evaluation system based on Adaptive Control. Methodology / approach - Will be demonstrating with an empirical example the rationality of the system. This example highlighted the problems and solutions for an effective performance appraisal that can be applied through a WEB platform, thus generating more than a performance evaluation system, but also a feedback system for starting or end work carrer, because it has the capacity for adapt the feedback on the level and area of the work. Findings - With the detailed construction of the model, the feasibility of the system was demonstrated in a methodical way, as well as it was evident the need for new research aimed at the popularization of the adaptive system. Contributions - The performance appraisal is of fundamental importance for the organizational output. However, it is observed that traditional systems fail to effectively measure performance, whether individual, group or organizational. The focus of this model presented is for co-participative and public organizations, however it can be used on private institutions as well.

Key-words: Performance Appraisal System; Adaptive systems; co-participation; Public administration; Performance management.
\end{abstract}

\section{Introdução}

A avaliação de desempenho é um tema complexo que deve levar em consideração a área, o nível 
de atuação e a situação temporal ou condições do ambiente. No serviço público, observa-se que esta ferramenta de gestão geralmente é utilizada para identificar necessidades de treinamento, progressões salariais ou avaliação no estágio probatório. Já na iniciativa privada, a avaliação de desempenho é mais usada para promoções, aumentos salariais ou como base para a estruturação de políticas salariais. De forma mais incisiva, Behn (2003, apud Martin et al. (2018)) afirma que existem oito principais propósitos na mensuração de desempenho, sendo eles: avaliar, controlar, orçar, motivar, promover, comemorar, aprender e melhorar.

Observa-se que o processo de feedback, nos modelos de avaliação, mostra-se importante para melhorar o desempenho dos colaboradores de qualquer organização, pois orienta na realização das tarefas e ações para um bom desempenho individual alinhado à missão, visão e objetivos organizacionais. Desta forma, é salutar que as organizações usem a avaliação de desempenho para comparar os resultados esperados com os obtidos; e para isto é necessário mensurar estes itens. Segundo Deming (1989), não se pode gerenciar o que não se pode medir.

Bourne et al. (2000) citam que o sistema de medição de desempenho requer desenvolvimento e revisão em diferentes níveis de mudança, tendo como foco: mecanismos efetivos para revisão e aprimoramento de metas e de padrões; processo de mediação individual, uma vez que as circunstâncias mudam; processo de revisão e reavaliação periódica do conjunto de medidas usadas na avaliação.

Todos estes itens, indicados por Bourne et al. (2000), podem ser alcançados com uma arquitetura de informação baseada em regras da área de Controle de Sistemas com seu subtópico chamado de Controle Adaptativo. Por exemplo, com uma pesquisa sistemática de boas práticas realizadas (como se fosse uma pesquisa de mercado - opinião, satisfação etc.), pode-se selecionar os melhores indicadores para serem usados na avaliação de desempenho e, se aplicada uma avaliação de qualidade a todas as áreas de uma organização com estes dados, há a possibilidade de alavancar a qualidade dos produtos, bens ou serviços fornecidos pela organização exatamente com um sistema de feedback.

Segundo Missel (2012), não fornecer o feedback é tão ruim quanto fornecê-lo de maneira equivocada. Nos dois casos, os efeitos podem ser devastadores, e é frequente a perda de talentos nas organizações por estas ações. Ressalta-se que empresas que praticam o feedback corporativo como ferramenta de gestão obtêm os melhores índices nas pesquisas de clima organizacional. Buscando minimizar este problema, Missel (2012) indica o uso do feedback 360 (trezentos e sessenta graus). A despeito de muitos estudos terem por objetivo aperfeiçoar este instrumento de gestão, deve ser destacado que ainda são encontrados alguns "gaps" que necessitam ser preenchidos para que a avaliação de desempenho possa adquirir maior efetividade e confiabilidade.

Evidencia-se que tradicionalmente os sistemas de avaliação de desempenho não usam ferramentas matemáticas sofisticadas, pois na área de recursos humanos (na administração), elas não são comuns, como em outras áreas como, por exemplo, engenharias, física, estatística, economia etc. Destacase que a matemática se mostra fundamental para se trabalhar com técnicas complexas que representam estudos ou representam comportamentos dinâmicos sobre sistemas mecânicos, elétricos, químicos, biológicos e até sociais. Por este motivo, entende-se que estudar a possibilidade de levar técnicas consagradas da engenharia de controle para a avaliação de desempenho apresenta-se relevante, especialmente em se tratando do serviço público brasileiro que possui tradicionalmente um desempenho pífio. Charbonnier-Voirine e Roussel (2012) citam trabalhos de 1999, 2000, 2002, 2006, que estudaram a avaliação de desempenho de forma adaptativa, cujas teorias, infelizmente, raro foram acompanhadas de recomendações de ferramentas de medição que abrangessem toda a gama de comportamentos desejados dentro das instituições.

Nota-se que caso o sistema de avaliação de desempenho seja participativo, este tem mais chances de sucesso. Sistemas participativos possuem uma tendência à seleção de atividades com maior precisão, pois todos os interessados (stakeholder) estão integrados ao processo, diferenciando-se do sistema tradicional, onde o gestor define as regras (ou indicadores) da avaliação. Além disso, quando as partes são consultadas, estas sentem-se na obrigação de participar, uma vez que propuseram os indicadores.

Desta forma, este artigo tem por objetivo evidenciar a importância de se desenvolver sistemas com bases matemáticas visando a uma maior efetividade na mensuração do desempenho, nos diversos níveis, bem com apresentar um modelo com base no controle adaptativo por meio de uma aplicação participativa. Com uma simulação considerável, busca-se demonstrar a viabilidade e a aplicabilidade de 
um sistema baseado em controle adaptativo no feedback e avaliação de desempenho em ambientes públicos ou participativos.

\section{Referencial Teórico}

\subsection{Avaliação de Desempenho}

De maneira objetiva, desde os primórdios do trabalho humano tem sido comum medir a quantidade e a qualidade do que foi feito. Assim, ao longo da história, a avaliação do desempenho tem acompanhado o trabalho; todavia, não tem sido de maneira formalizada. Só a partir do século XIX é que a avaliação de desempenho, instrumento elementar para os resultados organizacionais, passa a ser tratada de maneira formal (Edwars \& Ewen, 1996).

Para que a função de controle seja efetiva ao que se propõe, a avaliação de desempenho mostrase como a ferramenta gerencial ideal para este intento. Com a avaliação de desempenho, é possível compatibilizar o ideal e o real em termos de produtividade organizacional, pois é viabilizado o gerenciamento da organização. De acordo com Kaplan e Norton (1997), parafraseando Deming, o que não é medido não é gerenciado. Deste modo, a avaliação de desempenho permite medir quanto do planejamento foi executado e em que condições, adequando, desta forma, o plano à execução.

Desempenho é a realização efetiva de um trabalho (Ribeiro, 2017). Para Stoffel (1997), é toda ação que pode ser verificada e mensurada. Deve ser destacado que a avaliação de desempenho pode ser entendida como a comparação entre o que foi proposto - objetivos a atingir - e o que foi realizado - resultado atingido (Lucena, 1995).

Segundo Martin et al. (2018), os dois principais objetivos da mediação de desempenho possuem contradições, sendo elas o controle e a melhoria, estando estes itens conectados por outros quatro do processo de aprendizagem nas organizações, que são: reprodução de ações, regras orientadas, objetivos orientados e criatividade. Uma questão relevante é entender que o controle colabora com o processo de aprendizado, sendo necessário um trabalho rotineiro e eficiente, de forma a gerar um ambiente com ações criativas e de desenvolvimento.

Segundo Rosen et al. (2011), o gerenciamento de desempenho deve ser abrangente e representar aspectos tanto do indivíduo quanto do coletivo, pois o foco em um único nível, em detrimento de outro, pode levar à redução de desempenho. Esta afirmação dos autores pode ser matematicamente representada pela intersecção entre as necessidades, sendo que este processo de intersecção, matematicamente falando, exclui aquilo que não é comum às áreas.

Por fim, ressalta-se que existem diversos métodos e técnicas voltados à avaliação de desempenho, mas essa diversidade não elimina a ocorrência de vários erros de avaliação, conforme observa OliveiraCastro (1994).

\subsection{Problemas da Avaliação de Desempenho}

De acordo com Oliveira-Castro, Lima e Veiga (1996), na avaliação de desempenho, o fracasso se mostra como regra, sendo exceção os casos de sucesso. Para Ribeiro (2017), aplicar corretamente as técnicas de avaliação de desempenho é o maior problema, considerando a interferência de vários fatores, incluindo-se aí a subjetividade.

Além da subjetividade, o desconhecimento do processo, dos formulários e das pessoas que serão avaliadas evidencia-se como grande erro, que é ampliado e alavancado pela alta rotatividade de chefia, pois além de não se ter tempo para aprofundar o conhecimento do processo de avaliação, também não permite conhecer os avaliados (Oliveira-Castro, Lima \& Veiga, 1996; Ribeiro, 2017).

Dentre os problemas mais comuns decorrentes do não conhecimento dos avaliados e do processo de avaliação, destacam-se os efeitos halo, recenticidade, severidade, leniência e tendência central (Ribeiro, 2017), cada um gerando um problema diferente no processo de avaliação.

Deve ser destacado que Oliveira-Castro (1994) apresenta que o treinamento não resolve os efeitos de halo, leniência, severidade e tendência central, afirmando que o treinamento para eliminar erros é insuficiente para aumentar a eficácia da avaliação, podendo inclusive diminuir a precisão da avaliação.

Vasconcellos e Kruglianskas (1991), por seu turno, afirmam que, no processo de avaliar, ocorre uma barreira psicológica, posto que este é percebido como instrumento de penalização e premiação. 
Muitos avaliadores demonstram não gostar de avaliar pessoas, pois concebem a avaliação de desempenho como instrumento para dar resposta à área de recursos humanos (Lucena, 1995). Assim, os métodos de avaliação são escolhidos e só depois são definidos os objetivos em termos de trabalho. Segundo Lucena (1995), deveria ser o contrário. Como objetivos da avaliação de desempenho, Zedeck e Cascio (1982) destacam: 1) ação disciplinar, 2) desenvolvimento do avaliado, 3) promoção e 4) diagnóstico organizacional.

Apesar dos vários óbices em relação à utilização dos sistemas de avaliação de desempenho (Oliveira-Castro, Lima \& Veiga, 1996), são observados diversos indícios de que esse instrumento de gestão organizacional pode e deve ser utilizado como suporte no processo de gestão das organizações para que estas se tornem mais dinâmicas, flexíveis e eficientes. Por oportuno, deve ser notado que a discrepância de desempenho - caracterizada como a diferença entre o desempenho realizado e o desempenho esperado - pode indicar: 1) problemas de integração dos empregados; 2) problemas de supervisão; 3) ausência de motivação e 4) subaproveitamento de potencial.

Certamente existe um perigo quando se realiza uma simplificação no método de seleção dos indicadores para avaliar o desempenho. Uma norma ou regra simplista como critério de avaliação pode fazer com que os indivíduos da organização busquem metas de formas inapropriadas. 0 planejamento deve considerar não apenas uma fórmula robusta como também toda a entrada de dados, além da publicização destes dados posteriormente. Tal problema pode ser constatado com a adoção do Reconhecimento de Saberes e Competências (RSC) nas organizações de ensino. Com a criação de uma regra simplificada de avaliação como esta, o serviço público ficou mais caro e sem o esperado retorno eficaz à sociedade pagadora de impostos (Oliveira, 2017).

Nesta pesquisa interdisciplinar, propõe-se o uso de ferramentas clássicas da engenharia de controle como forma de organizar e fazer funcionar a avaliação de desempenho dos recursos humanos das organizações, em especial das instituições públicas, ou sem fins lucrativos, que tem uma orientação para atividades coparticipativas. Na figura 1 está representada a proposta de Megginson, Mosley e Pietri (1998) com a região de aplicação desta proposta.

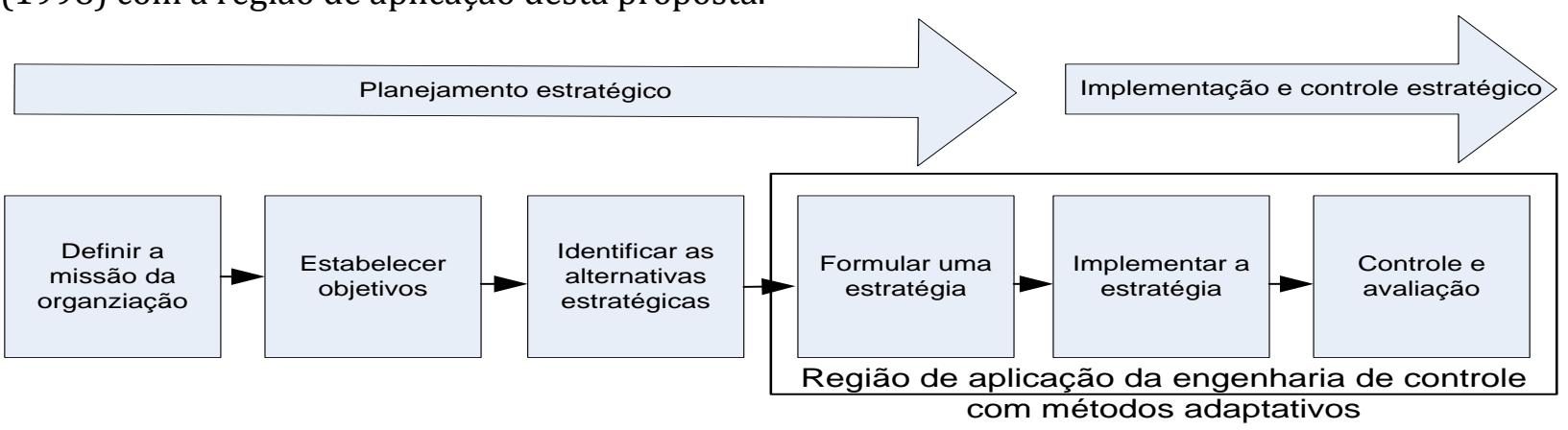

Figura 1. Planejamento estratégico e a região de implementação de algoritmos adaptativos de avaliação de desempenho.

Fonte: Megginson, Mosley e Pietri (1998), com adaptações.

Organizações, públicas ou privadas, sofrem alterações quando uma nova geração troca os hábitos da anterior. Muitas vezes, não só as atividades internas da empresa mudam, mas as externas também, principalmente com a mudança dos hábitos de consumo e necessidades dos seus clientes. Outras vezes, as mudanças são impostas por culturas de novos clientes ou por tecnologias emergentes. Para estes casos, a necessidade de adaptação faz parte do processo de manutenção da organização. Estas adaptações podem gerar novos comportamentos de desempenho sempre com foco na melhoria, mas em alguns casos podem piorar o que já existia.

Segundo Charbonnier-Voirine e Roussel (2012), a capacidade de adaptação refere-se à habilidade de um indivíduo em alterar seu comportamento para atender às demandas de um novo ambiente, ou seja, requer que os funcionários se adaptem rápida e facilmente e tomem decisões diante da incerteza e ambiguidade inerentes à situação. De forma complementar, o desempenho adaptativo bem-sucedido implica que os funcionários são capazes de lidar eficientemente com situações de trabalho incertas e com imprevistos que podem, por exemplo, resultar em reestruturação organizacional, mudança de prioridades ou menor disponibilidade de recursos. 
Como orientação histórica, Charbonnier-Voirine e Roussel (2012) indicam que Hesketh e Neal (1999) foram os primeiros a propor o conceito de desempenho adaptativo, enquanto Murphy e Jackson (1999) se referiram à flexibilidade de papéis (atuações e ações) e London e Mone (1999) destacaram a importância das capacidades dos funcionários para reagir e gerenciar novas experiências

A estratégia de avaliação proposta neste artigo considera que, se todos os trabalhadores estiverem realizando seu trabalho em um alto grau de desempenho, baseado em indicadores bem selecionados, então a empresa estará saudável.

\subsection{Controle Adaptativo}

0 projeto de engenharia, na forma de uma arquitetura de informação que contenha as qualidades indicadas nos sistemas coparticipativos, pode ter como ponto de partida as técnicas de Controle Adaptativo oriundo da Engenharia de Controle de Sistemas. Para Tao (2014), o controle adaptativo possui uma metodologia capaz de trabalhar com incertezas dos sistemas, mantendo o desempenho desejado, pois ele altera internamente sua forma ou estrutura para se adequar ao ambiente. Aström e Wittenmark (2008) afirmam que um controlador com características adaptativas deve mudar seus parâmetros internos mediante um mecanismo de ajuste que deve verificar o melhor desempenho ou funcionalidade do sistema que está sob controle.

A técnica de Controle Adaptativo pode ser utilizada para seleção dos indicadores de desempenho de forma coparticipativa. Assim, mudam-se, de forma automática, os indicadores de avaliação de desempenho (pessoal) com o propósito de ajudar a organização a melhorar sua adaptação, flexibilidade e descentralização na tomada de decisão. Entretanto, cuidados devem ser tomados, pois indicadores de desempenho selecionados por profissionais não especializados podem gerar erros grosseiros na avaliação (especialmente no serviço público brasileiro) sem uma técnica robusta de controle dos dados coletados e revisão constante na implementação dos indicadores.

Para descrever e deixar evidentes a extensão e as possibilidades do controle adaptativo, podem ser citadas suas aplicações clássicas, como: orientação de mísseis, naves e navios, controle de movimento em robôs, máquinas ferramenta, colunas de destilação, além de outras. Para todos estes casos, pode-se controlar os sistemas complexos, sem o conhecimento de todas as variáveis, e mesmo assim manter suas funcionalidades com bons índices de desempenho (Aström \& Wittenmark, 2008).

Para o processo de avaliação de desempenho adaptativo, é necessário um conjunto de adaptações para se transformar sinais físicos (mecânicos, elétricos, transmissão de dados etc.) em elementos equivalentes aos sistemas de comunicação e referência usados por seres humanos em organizações e instituições de trabalho. Para este caso, propõe-se que se utilizem os indicadores como sendo as "melhores práticas" para fazer com que o sistema de avaliação de desempenho também seja um sistema de orientação profissional (coaching), ou seja, que não se mensure apenas o que foi ou será alcançado pelos empregados ou servidores, mas também como será alcançado. Com isso, a velocidade de aprendizado de qualquer novo funcionário da empresa terá uma curva de crescimento incrementada.

Todavia, ressalta-se a questão de o porquê ensinar a trabalhar. Neste sentido, Missel (2012) constata que as pessoas são contratadas pelas suas habilidades técnicas e demitidas por sua dificuldade de comunicação e comportamentos, tornando as características de personalidade essenciais no processo de seleção, promoção e até de demissão. É fundamental levar em consideração que no serviço público brasileiro (na prática) as pessoas não são demitidas. Exceção à regra, quando são condenadas judicialmente por algum crime cometido, mas quase ninguém é exonerado por ser incompetente ou agir de forma pífia nas suas atividades diárias, muito diferente do serviço privado. Esta constatação reforça o artigo de Galindo (2017) que fala da ineficiência do serviço público.

Para este processo de conversão da teoria de controle adaptativo para uma arquitetura de informação, orientação e avaliação de desempenho, devem ser abordados os itens básicos necessários no projeto de um controlador clássico para depois se proporem as adaptações ao ambiente corporativo. Seguindo as pesquisas de Aström e Wittenmark (2008), na construção de um controlador adaptativo, é necessário passar pelos seguintes passos: 1) definir o comportamento desejado dentro de um laço de controle (closed-loop); 2) determinar uma lei de controle adequada para o ajuste automático dos parâmetros do controlador; 3) encontrar o mecanismo de "gatilho" para o ajuste de parâmetros; e 4) implementar o algoritmo da lei de controle. 
Como característica técnica fundamental para selecionar o controle adaptativo conforme uma técnica interessante para o uso na avaliação de desempenho de pessoas, pode-se citar o fato de que mesmo quando os parâmetros não convergirem para um valor fixo, a malha de controle fará com que o sistema convirja para o valor desejado (resultado final), ou seja, as mudanças internas controladas geram um comportamento externo que converge para o valor desejado. Além disto, outras características do controle adaptativo (Aström e Wittenmark, 2008) são apresentadas, como: o modelo matemático do sistema não necessita ser preciso, ou seja, é robusto a pequenos erros; tem capacidade de ser aplicado a sistemas não lineares e variantes ao longo do tempo; pode ser aplicado em tempo real, ou seja, em uma velocidade alta que possibilita ações on-line e dispõe de adaptabilidade a condições adversas, ou seja, não previstas.

Como a arquitetura proposta trabalha com seres humanos, é fundamental usar a estatística como interface entre os dados encontrados entre pessoas com visões diferentes e os dados otimizados para uso final como critérios para avaliação de desempenho. Ressalta-se que existe diferença técnica entre sistemas robustos, inteligentes e adaptativos. Para alguém fora da área de engenharia de controle, isto pode parecer a mesma coisa, mas elas são bem diferentes no momento dos cálculos e da implementação física e lógica (Oliveira, Deschamps \& Lima, 2016).

\section{Metodologia}

Partindo-se do princípio de que um sistema controlado por uma técnica de controle adaptativo possui uma estrutura rígida (determinada) com capacidade de mudar automaticamente seus parâmetros mediante um algoritmo de ajuste, isto quer dizer que ele é capaz de trabalhar sobre algumas incertezas do sistema. Uma vez que quem executa as tarefas nas organizações são as pessoas, o comportamento delas é fator preponderante no desempenho da instituição, então elas serão os controladores que devem ter seus "parâmetros" ajustados, ou seja, sua prática.

A proposta deste trabalho é apresentar uma nova relação entre um problema clássico e complexo, usando uma metodologia não convencional para a área. É sabido que os trabalhadores devem possuir critérios de avaliação de desempenho para desenvolver adequadamente seu trabalho. Para os sistemas de controle adaptativo, também é necessário o ajuste e o desenvolvimento de um sistema de avaliação de desempenho na forma de um mecanismo que diga quando os parâmetros do sistema de controle devem ser alterados. Analisando-se estes dois itens, chega-se à conclusão de que é possível criar um sistema de avaliação de desempenho humano com as regras do controle adaptativo. Graficamente, esta relação é demonstrada pela figura 2 .

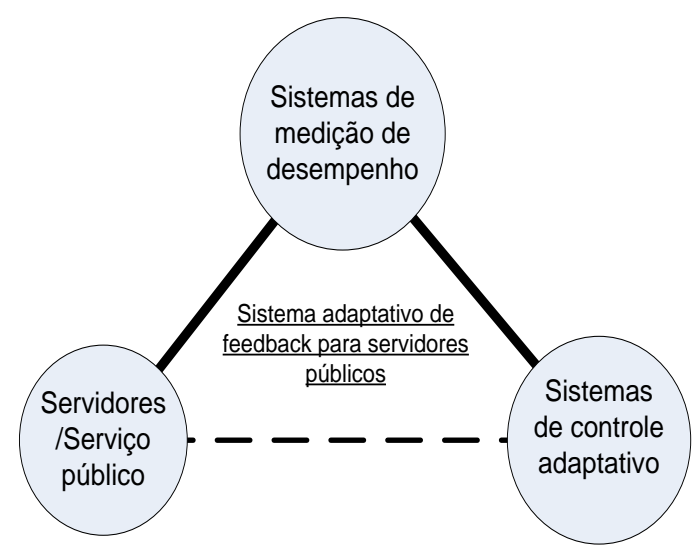

Figura 2. Relação entre a medição de sistemas adaptativos e medição de desempenho.

Fonte: Os autores.

Com base nas pesquisas de Controle Adaptativo, sabe-se que o controle com autossintonia deve possuir dois laços de informação (feedback loops). 0 laço interno, também chamado de malha interna, contém o método clássico de controle com parâmetros que variam ao longo do tempo. Já o laço externo (ou malha externa) é formado por um algoritmo de identificação do comportamento do sistema a ser controlado e um algoritmo secundário que ajusta os parâmetros do controlador com base nestes dados 
anteriormente encontrados, ou seja, na identificação. 0 processo contínuo e sistemático transforma a arquitetura de autossintonia em uma arquitetura adaptativa, ou seja, a seleção dos parâmetros não acontece em uma única vez, mas sempre que for reconhecida a necessidade de ajuste pelo algoritmo.

A arquitetura básica de um sistema de Controle Adaptativo ajustado para um sistema de avaliação de desempenho é apresentada na Figura 3. Para a revisão da teoria geral de controle adaptativo em sistemas eletromecânicos, é recomendada a leitura de Aström e Wittenmark (2008), sendo os blocos básicos da figura abaixo apresentados já dentro do contexto da avaliação de desempenho adaptativo para seres humanos.

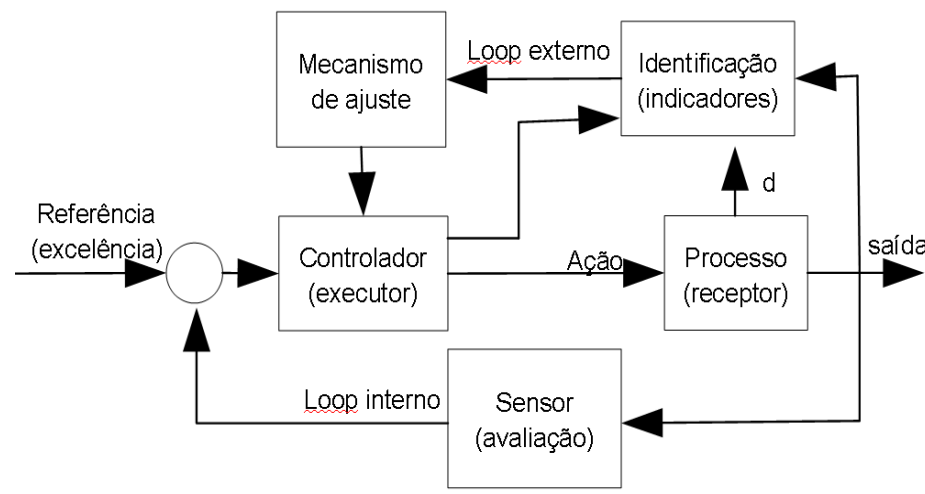

Figura 3. Controle adaptativo ajustado para um sistema de avaliação de desempenho.

Fonte: Oliveira, Deschamps e Lima (2016), com adaptações.

A Figura 3 apresenta o diagrama básico de controle adaptativo. Para a sua aplicação ao caso da avaliação de desempenho no ambiente de trabalho organizacional coparticipativo, propõem-se as seguintes alterações nos sinais e blocos:

a) Referência: este sinal indica o nível a ser alcançado, que deve ser o máximo possível dentro de uma meta definida. Propõem-se que, para seres humanos, a meta deve ser de, no mínimo, 70\% para ser entendida como realizada de forma eficaz. Este valor é oriundo da tensão eficaz de sistemas elétricos de corrente alternada, também conhecida como tensão RMS (Root Mean Square);

b) Controlador: este bloco simboliza quem executará as ações na instituição e precisará do feedback. Pode ser qualquer profissional que esteja procurando realizar um bom trabalho, por exemplo, um diretor ou um coordenador;

c) Sensores: são os elementos indicadores (mensuradores) que apresentam a eficiência das ações tomadas. É fundamental que técnicas apuradas sejam aplicadas neste contexto, pois caso contrário erros graves podem ocorrer. Lembrando que indicadores e mensuradores inapropriados levam a ações inapropriadas;

d) Mecanismo de identificação: é o uso de técnicas de otimização e estatística para a seleção das atividades que merecem ser avaliadas. É fundamental definir prioridades, caso contrário, um processamento de busca de indicadores pode chegar a conclusões absurdas, tais como dizer que a apresentação de um pôster em um congresso municipal tem valor (peso ou importância) superior à publicação em uma revista científica internacional de alto impacto.

e) Mecanismo de ajuste: os dados alcançados pelo mecanismo de identificação são apresentados no formato de critérios de desempenho que dão orientação ao desenvolvimento do trabalho por parte do executor das tarefas (trabalhadores). Estes critérios serão usados também na avaliação de desempenho, ou seja, antes da avaliação, o executor (colaborador da organização) já sabe quais serão os critérios de avaliação daquele ano ou período, ou seja, tudo explicitado, com previsão e planejamento.

f) Processo ou receptor: descreve o grupo que recebe o resultado das ações realizadas pelo colaborador que executa as ações (na Figura 3 representado pelo processo). Este grupo participará da avaliação de desempenho de duas formas: a primeira, por meio da avaliação final do executor das atividades e, a segunda, fazendo parte da seleção dos indicadores de avaliação de desempenho. Este último item é fundamental para que o próprio executor não crie sozinho os indicadores, coisa que poderia levar o sistema de avaliação a uma falha grave, pois os indicadores selecionados 
poderiam ter uma orientação inadequada.

\section{Proposta para um Sistema de Avaliação de Desempenho Adaptativo}

Dentro do mecanismo de identificação dos indicadores de avaliação, é necessário impor regras estatísticas, aqui chamadas de filtros humanos, pois o algoritmo envolve pessoas. Sabendo-se que o sistema de indicadores deve aceitar propostas dos envolvidos no processo (método do feedback 360ㅇ) e que estas propostas podem estar distorcidas tanto por parte dos executores como pelos receptores, é fundamental trabalhar com a capacidade de exclusão daquilo que não é considerado como adequado por ambos, ou ao menos de baixa prioridade.

A proposta matemática que resolve este problema é o uso da Interseção. Na figura 4, é apresentada graficamente esta relação. Matematicamente, ela pode ser apresentada pela expressão $\mathrm{P}=\mathrm{A} \cap \mathrm{B} \cap \mathrm{C}$, onde "P" é o grupo de indicadores selecionados; "A" são os indicadores propostos pelo grupo A; "B" são os indicadores propostos pelos grupos B e "C" são os indicadores propostos pelo grupo C, etc. Desta forma, a possibilidade do uso de indicadores mal escritos, mal interpretados ou, até mesmo, mal-intencionados cai a valores próximos de zero (Oliveira, Deschamps \& Lima, 2016).

Exemplificando os grupos envolvidos no processo acima, o grupo A poderia ser da alta gerência da instituição, o grupo B dos executores das tarefas e o grupo C dos receptores do trabalho (clientes). Dentro de uma instituição de ensino, como exemplo hipotético, o grupo A pode ser o Ministério da Educação (MEC) ou pedagogos institucionais, o grupo B, os professores e o grupo C, os alunos.

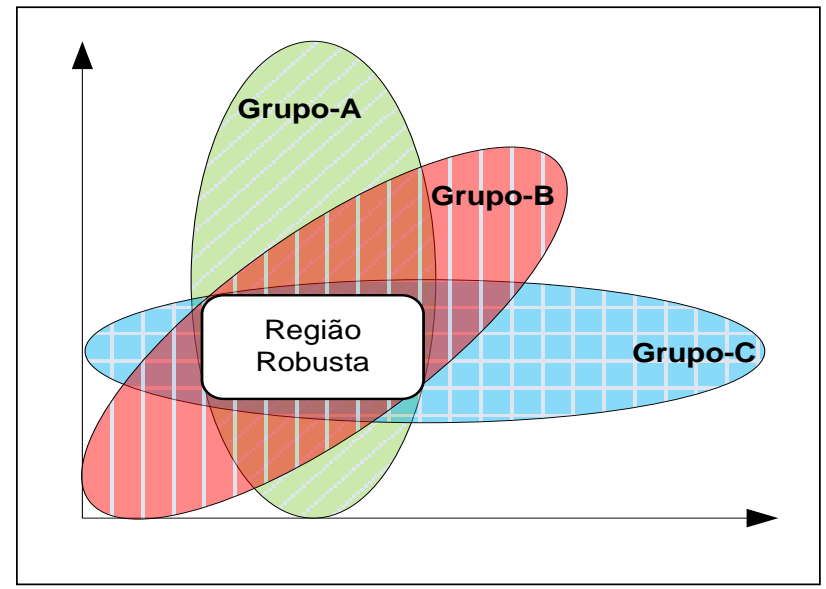

Figura 4. Exemplo gráfico de uma seleção robusta de indicadores de desempenho participativa.

Fonte: Os autores.

Este processo de seleção dos melhores indicadores se enquadra como um sistema de otimização, uma vez que a otimização é o processo de se obter o melhor resultado possível baseado em determinadas restrições e circunstâncias, ao final sempre minimizando o esforço necessário e maximizando o benefício alcançado (Rao, 2009).

Na sequência abaixo, de forma ilustrativa, é proposta a Lei de Controle, ou seja, o mecanismo de ajuste de parâmetros para encontrar os melhores indicadores para a avaliação de desempenho no serviço público ou instituições coparticipativas. A Lei de Controle proposta neste artigo é apresentada na forma de um algoritmo, cujos passos básicos são:

1) iniciar o processo com data previamente estipulada ("gatilho" de mudanças);

2) realizar uma pesquisa junto ao "grupo A" e ranquear os indicadores (boas práticas) mais importantes para este grupo;// por exemplo: coordenadores;

3) realizar uma pesquisa junto ao "grupo B" e ranquear os indicadores (boas práticas) mais importantes para este grupo;//por exemplo: subordinados;

4) realizar uma pesquisa junto ao "grupo C" e ranquear os indicadores (boas práticas) mais importantes para este grupo;// por exemplo: coordenadores de outras instituições, mas da mesma área de atuação, direção geral ou especialistas da área;

5) encontrar os melhores indicadores para este ano com a função: $P_{n}(t)=M A X_{n}(A \frown B \frown C)$; 
6) definir a quantidade mínima de indicadores (n) e tornar público o novo conjunto de indicadores para a avaliação de desempenho $P_{n}(t)$ e

7) finalizar o mecanismo de ajuste. Tendo em mãos um conjunto de indicadores (P) para a avaliação de desempenho, o próximo passo é usá-los na mensuração de desempenho, que com esta metodologia se mostra robusta por usar informações de mais de uma área, podendo chegar a uma avaliação $360^{\circ}$.

Evidencia-se que toda vez que for usado o termo "indicadores", este pode ser interpretado como variáveis, ações ou, ainda, boas práticas. Estes itens mudarão devido a, no mínimo, três dimensões:

1) área de atuação da atividade desenvolvida;

2) nível de atuação (como, por exemplo, básico, médio e avançado) e

3) maturidade institucional, em que, provavelmente, "pequenos" problemas já foram resolvidos e agora a instituição consegue focar em atividades mais complexas.

Seguindo pela linha histórica de que o homem criou ferramentas para auxílio em suas tarefas, o método aqui proposto pode ser aplicado como ferramenta WEB que também melhorará o desempenho das ações tomadas, aumentando a produtividade. Todavia, é importante que esta mudança seja sistemática para que erros possam ser corrigidos em tempo. Com base nisso, as técnicas de Controle Adaptativo são interessantes para controlar a mudança de forma sistemática, coparticipativa e de forma orientada (coaching) mesmo quando o ambiente impõe certas perturbações que podem desestabilizar a estrutura de otimização proposta.

Para demonstrar numericamente o que foi dito até este momento, no tópico seguinte serão apresentadas, de forma prática, as etapas a serem percorridas para aplicar um sistema de avaliação de desempenho adaptativo levando em consideração dois agentes das organizações: os chefes e os empregados.

\subsection{Etapas para um Sistema de Avaliação de Desempenho Adaptativo}

Com vistas à exemplificação de um sistema de avaliação de desempenho adaptativo, será demonstrada a aplicação do método, em um caso hipotético, para a avaliação de desempenho de um coordenador de uma equipe administrativa de uma instituição prestadora de serviço, como por exemplo um coordenador administrativo. Cada um dos indicadores apresentados possui a nomenclatura Qx, onde o "Q" é referente à "Questão" usada na avaliação de desempenho e o índice "x" é o número do item, como por exemplo: Q1, Q2, Q3 etc.

A princípio, existem duas formas de se fazer as perguntas da pesquisa (Qx). Na primeira, a própria instituição começa com uma lista definida pela gestão, sendo os itens mais importantes ranqueados para uso. Na segunda, a lista de itens a serem selecionados pode vir de uma revisão da literatura, que dispõe de um banco de dados interessante para se iniciar, a saber: os indicadores existentes em coleções de Indicadores Chave de Desempenho (Key Performance Indicators - KPI). Existe mais de uma coletânea de indicadores que podem ser usados nas mais diversas áreas, ficando à disposição dos avaliadores a sua inserção e uso no sistema de avaliação de desempenho e feedback (Parmenter, 2012; Parmenter, 2010; Marr, 2012; The KPI institute, 2013). É importante ressaltar que se apenas quem será avaliado for o seletor dos indicadores da avaliação, estes podem estar deturpados por um processo de "legislar em causa própria".

Na Tabela 1, que contém os dados iniciais da simulação, é apresentado um conjunto de trinta questões definidas como boas práticas que devem ser seguidas anualmente pelo coordenador, ainda que se possam usar apenas dez ou quinze para a avaliação de desempenho. Com esta arquitetura, as questões (boas práticas - Qx) mais importantes para a avaliação vão ser encontradas com definição de prioridade, melhorando, desta forma, a qualidade da instituição, pois os coordenadores sabem quais são as boas práticas a serem executadas no ambiente de trabalho.

0 período de simulação completa foi de sete anos, desde 2010 até 2017, conforme a Figura 5, todavia são muitos dados e por isso algumas adaptações gráficas foram feitas. A Tabela 1 apresenta os dados de simulação entre 2010 e 2012. Como a apresentação de todos os dados é inviável, para facilitar a visualização, a simulação foi feita com apenas dois grupos, de coordenadores e de subordinados.

Os dados de 2010 foram de uma pré-pesquisa que indicou as boas práticas em condições iniciais 
(genéricas), não necessariamente corretas e indicadas pela bibliografia da área ou pela direção da instituição. No ano de 2011, os coordenadores (co) e os subordinados (su) fazem suas considerações sobre a ordem de importância e isto gera o posicionamento interno (inter); no passo seguinte, os dados são aplicados com um fator inercial, ou seja, no rank, uma parte do valor final (posição ou prioridade) é dada pela pesquisa do ano corrente e outra, do ano anterior. Com isso, minimizam-se variações bruscas nos indicadores. No último passo, os dados são normalizados (de 0\% a 100\%) para a seleção dos dez indicadores mais importantes para a aplicação na avaliação de desempenho. Este procedimento foi realizado nos demais anos até 2017.

Tabela 1 - Dados da simulação para os anos de 2011 e 2012.

\begin{tabular}{|c|c|c|c|c|c|c|c|c|c|c|c|}
\hline \multirow{2}{*}{\multicolumn{2}{|c|}{$\begin{array}{l}2010 \\
\text { Inicial }\end{array}$}} & \multicolumn{5}{|c|}{2011} & \multicolumn{5}{|l|}{2012} \\
\hline & & Co & Su & inter & Rank & Ordem & Co & Su & Inter & Rank & Ordem \\
\hline Q1 & 100,0 & 90,3 & 77,4 & 86,4 & 90,5 & 99,7 & 96,3 & 64,3 & 86,7 & 90,6 & 99,7 \\
\hline Q2 & 97,5 & 90,2 & 82,4 & 87,9 & 90,8 & 100,0 & 96,3 & 65,0 & 86,9 & 90,8 & 100,0 \\
\hline Q3 & 95,0 & 81,6 & 76,5 & 80,1 & 84,5 & 93,1 & 93,7 & 67,9 & 85,9 & 88,1 & 97,0 \\
\hline Q4 & 92,5 & 88,6 & 86,9 & 88,1 & 89,4 & 98,5 & 91,1 & 68,4 & 84,3 & 88,5 & 97,5 \\
\hline Q5 & 90,0 & 20,0 & 81,7 & 38,5 & 54,0 & 59,4 & 30,0 & 69,3 & 41,8 & 47,1 & 51,8 \\
\hline Q6 & 87,5 & 85,5 & 74,7 & 82,3 & 83,8 & 92,3 & 93,7 & 70,7 & 86,8 & 88,4 & 97,4 \\
\hline Q7 & 85,0 & 86,8 & 78,5 & 84,3 & 84,5 & 93,1 & 95,3 & 74,3 & 89,0 & 90,2 & 99,3 \\
\hline Q8 & 82,5 & 85,4 & 83,3 & 84,8 & 84,1 & 92,6 & 91,3 & 77,0 & 87,0 & 88,7 & 97,6 \\
\hline Q9 & 80,0 & 96,0 & 30,0 & 76,2 & 77,3 & 85,2 & 97,1 & 20,0 & 74,0 & 77,3 & 85,1 \\
\hline Q10 & 77,5 & 79,3 & 78,0 & 78,9 & 78,5 & 86,5 & 92,2 & 79,5 & 88,4 & 87,8 & 96,6 \\
\hline Q11 & 75,0 & 77,2 & 55,8 & 70,8 & 72,0 & 79,4 & 90,3 & 79,7 & 87,1 & 84,8 & 93,3 \\
\hline Q12 & 72,5 & 78,6 & 55,9 & 71,8 & 72,0 & 79,3 & 87,9 & 80,0 & 85,5 & 83,7 & 92,1 \\
\hline Q13 & 70,0 & 86,6 & 69,8 & 81,5 & 78,1 & 86,0 & 92,8 & 80,3 & 89,0 & 88,1 & 97,0 \\
\hline Q14 & 67,5 & 78,5 & 73,1 & 76,9 & 74,1 & 81,6 & 89,1 & 80,6 & 86,5 & 85,0 & 93,6 \\
\hline Q15 & 65,0 & 78,1 & 67,9 & 75,0 & 72,0 & 79,3 & 50,0 & 81,5 & 59,5 & 65,4 & 72,0 \\
\hline Q16 & 62,5 & 73,1 & 60,1 & 69,2 & 67,2 & 74,0 & 85,0 & 83,3 & 84,5 & 81,3 & 89,5 \\
\hline Q17 & 60,0 & 80,0 & 69,5 & 76,9 & 71,8 & 79,1 & 55,0 & 83,7 & 63,6 & 68,3 & 75,1 \\
\hline Q18 & 57,5 & 72,8 & 63,1 & 69,9 & 66,2 & 72,9 & 80,0 & 83,9 & 81,2 & 78,7 & 86,6 \\
\hline Q19 & 55,0 & 72,7 & 61,6 & 69,4 & 65,1 & 71,7 & 60,0 & 89,3 & 68,8 & 69,7 & 76,7 \\
\hline Q20 & 52,5 & 76,1 & 71,7 & 74,8 & 68,1 & 75,0 & 75,0 & 89,5 & 79,4 & 78,1 & 85,9 \\
\hline Q21 & 50,0 & 72,8 & 61,6 & 69,5 & 63,6 & 70,1 & 65,0 & 90,4 & 72,6 & 71,9 & 79,1 \\
\hline Q22 & 47,5 & 71,9 & 60,6 & 68,5 & 62,2 & 68,5 & 70,0 & 91,6 & 76,5 & 74,1 & 81,6 \\
\hline Q23 & 45,0 & 70,9 & 59,5 & 67,5 & 60,8 & 66,9 & 70,0 & 92,8 & 76,8 & 73,9 & 81,3 \\
\hline Q24 & 42,5 & 70,0 & 58,5 & 66,5 & 59,3 & 65,3 & 66,0 & 94,1 & 74,4 & 71,7 & 78,9 \\
\hline Q25 & 40,0 & 69,0 & 57,4 & 65,5 & 57,9 & 63,8 & 88,0 & 95,3 & 90,2 & 82,3 & 90,6 \\
\hline Q26 & 37,5 & 68,1 & 56,3 & 64,6 & 56,4 & 62,2 & 77,0 & 96,6 & 82,9 & 76,7 & 84,4 \\
\hline Q27 & 35,0 & 67,1 & 55,3 & 63,6 & 55,0 & 60,6 & 66,0 & 97,8 & 75,5 & 71,1 & 78,2 \\
\hline Q28 & 32,5 & 66,2 & 54,2 & 62,6 & 53,6 & 59,0 & 55,0 & 99,0 & 68,2 & 65,5 & 72,0 \\
\hline Q29 & 30,0 & 65,3 & 53,2 & 61,6 & 52,1 & 57,4 & 44,0 & 100,3 & 60,9 & 59,8 & 65,9 \\
\hline Q30 & 27,5 & 64,3 & 52,1 & 60,6 & 50,7 & 55,8 & 33,0 & 101,5 & 53,6 & 54,2 & 59,7 \\
\hline
\end{tabular}

Fonte: Os autores.

Legenda: co: coordenadores; su: subordinados; inter: interno; Rank: cálculo interno; Ordem: resultado final com aplicação de inércia.

Para demonstrar a robustez dos cálculos, os itens Q5 e Q9 foram deixados baixos, como se fossem votados como itens impróprios ou insignificantes para a avaliação de desempenho. Ao longo das avaliações, eles vão decaindo, ou seja, questões inapropriadas propostas por qualquer grupo não se mantêm para uso nas avaliações de desempenho. Em uma discussão mais ampla, o método proposto possui uma beleza intrínseca, que é o expurgo da corrupção, quando o indicador que não for bom para ambos os grupos será excluído. Representando matematicamente, a corrupção é a ação que é boa para um grupo, em detrimento de outro. Essa característica é interessante para o serviço público.

Na figura 5, são apresentados, de forma gráfica, todos os trinta indicadores ao longo de oito anos. No ano de 2014, foi simulado um distúrbio, em que uma variação drástica de políticas de gestão, normas e até implantação de uma nova célula de trabalho desestabilizaram pontualmente os critérios usados na avaliação, contudo, como o algoritmo é adaptativo, os indicadores voltaram a se estabilizar nos próximos anos da pesquisa para seleção dos indicadores para a avaliação de desempenho.

Baseados no gráfico da simulação (Figura 5), os dados convergiram rapidamente. Em apenas três anos de aplicação do sistema de avaliação de desempenho adaptativo, as boas práticas mais importantes convergiram para a região de uso (no topo da figura), ou seja, valores que as definem como importantes 
para uso na avaliação de desempenho. Em 2014, foi inserida propositalmente uma perturbação no sistema de identificação na forma de um grupo que fizesse imposições errôneas, mas o sistema se recuperou e conseguiu mudar os dados e convergir para valores aceitáveis nos anos seguintes $(2015,2016$ e 2017).

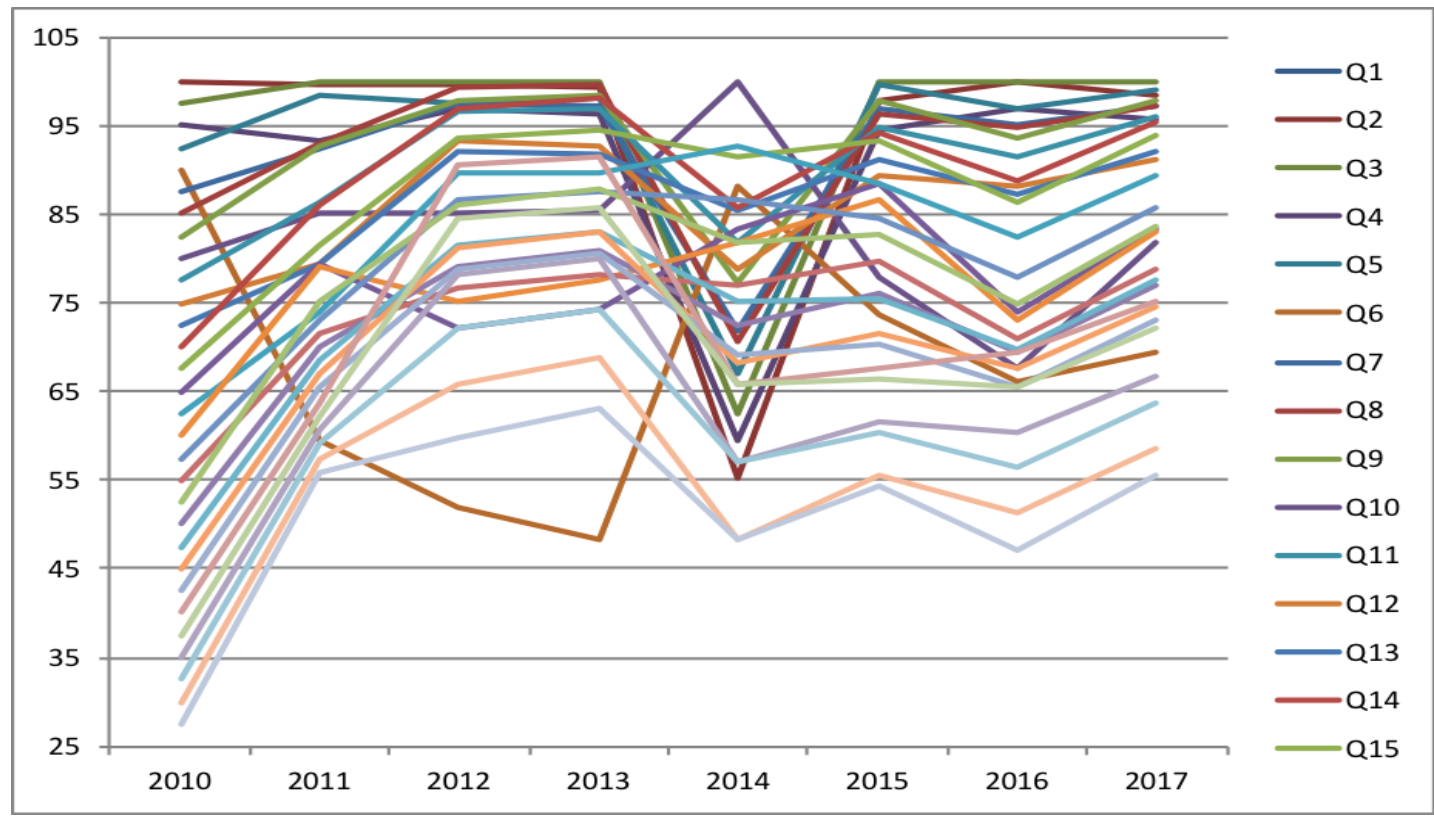

Figura 5. Exemplo de indicadores sendo selecionados por uma arquitetura adaptativa. Os itens de maior aceitação, no topo da figura, são os escolhidos para a avaliação de desempenho.

Fontes: Os autores.

Analisando a dinâmica no processo de identificação dos indicadores mais adequados para avaliação de desempenho (Figura 5), entende-se que o sistema está controlado e otimizado. Esta mesma implementação de arquitetura deve ser engenhada para cada área da organização. Desta forma, geram-se malhas de controle distribuídas com ações especializadas por área, ao mesmo tempo em que se evita o uso de regras de avaliação pífias e insignificantes criadas para agradar determinados grupos. Quanto mais distribuído for o controle, menor será a possibilidade de um erro sistêmico que leve a falhas funcionais. Analisando o comportamento dos indicadores da figura 5 ao longo do tempo, tem-se os seguintes resultados:

a) Q1, que inicialmente foi definida como sendo o item mais importante, se estabilizou na terceira posição;

b) Q2 se estabilizou em primeiro lugar;

c) Q3 se estabilizou no oitavo lugar, ou seja, caiu significativamente sua prioridade entre o início e a estabilização;

d) Q4 foi decaindo até o ano de 2014 e, depois disso, voltou a ser selecionado como um item importante, estabilizando-se no final como segundo item mais importante;

e) Q5, o elemento definido como errôneo, caiu rapidamente na pesquisa, retornou no período conturbado definido (2014), mas voltou a sair da região de seleção, ou seja, sua prioridade é tão baixa que ele não recebeu pontuação o suficiente para permanecer entre os selecionados para uso;

f) Q6 sofreu deslocamentos devido à dinâmica do sistema, mas acabou voltando para o seu local inicial;

g) Q7 subiu rapidamente até 2013, no ano da perturbação ele saiu da avaliação, mas nos anos seguintes voltou a ser usado. Se a simulação fosse realizada por mais alguns anos, provavelmente chegaria a ficar entre os cinco primeiros;

h) Q8 teve um comportamento parecido com Q6. Este indicador é estável, pois foi usado em todos os anos normais da pesquisa, o quer dizer que ele é definido como importante pelos grupos pesquisados e que selecionam os indicadores;

i) Q9 também foi um indicador errôneo. Ele decaiu rapidamente durante os ciclos de identificação, voltou no ano da perturbação, mas voltou a cair da lista de indicadores devido a sua ineficiência vista pelos dois grupos pesquisados e 
j) Q10 é o indicador mais estável da pesquisa. Apesar de estar próximo da região de corte para não ser usado como critério de avaliação, ele sempre se manteve entre os dez primeiros, ou seja, é um indicador funcional e reconhecido por todos. Mesmo não sendo o indicador mais importante, ele é importante na avaliação de desempenho.

Como a quantidade de dados é grande, fica mais fácil a visualização se forem apresentadas apenas as dez (10) boas práticas mais importantes encontrados após cada ciclo de pesquisa com as partes envolvidas. Na Tabela 2, tem-se os valores numéricos e respectivos indicadores encontrados para a avaliação de desempenho conforme a Figura 5.

Tabela 2 - Rank das questões mais importantes na avaliação de desempenho coparticipativo

\begin{tabular}{c|c|c|c|c|c|c|c|c}
\hline no & $\mathbf{2 0 1 0}$ & $\mathbf{2 0 1 1}$ & $\mathbf{2 0 1 2}$ & $\mathbf{2 0 1 3}$ & $\mathbf{2 0 1 4}$ & $\mathbf{2 0 1 5}$ & $\mathbf{2 0 1 6}$ & $\mathbf{2 0 1 7}$ \\
\hline $\mathbf{1}$ & $\mathrm{Q} 1$ & $\mathrm{Q} 2$ & $\mathrm{Q} 2$ & $\mathrm{Q} 2$ & $\mathrm{Q} 9$ & $\mathrm{Q} 2$ & $\mathrm{Q} 1$ & $\mathrm{Q} 2$ \\
$\mathbf{2}$ & $\mathrm{Q} 2$ & $\mathrm{Q} 1$ & $\mathrm{Q} 1$ & $\mathrm{Q} 7$ & $\mathrm{Q} 16$ & $\mathrm{Q} 4$ & $\mathrm{Q} 2$ & $\mathrm{Q} 4$ \\
$\mathbf{3}$ & $\mathrm{Q} 3$ & $\mathrm{Q} 4$ & $\mathrm{Q} 7$ & $\mathrm{Q} 1$ & $\mathrm{Q} 14$ & $\mathrm{Q} 1$ & $\mathrm{Q} 4$ & $\mathrm{Q} 1$ \\
$\mathbf{4}$ & $\mathrm{Q} 4$ & $\mathrm{Q} 3$ & $\mathrm{Q} 8$ & $\mathrm{Q} 8$ & $\mathrm{Q} 5$ & $\mathrm{Q} 8$ & $\mathrm{Q} 3$ & $\mathrm{Q} 8$ \\
$\mathbf{5}$ & $\mathrm{Q} 5$ & $\mathrm{Q} 7$ & $\mathrm{Q} 4$ & $\mathrm{Q} 13$ & $\mathrm{Q} 18$ & $\mathrm{Q} 6$ & $\mathrm{Q} 6$ & $\mathrm{Q} 7$ \\
$\mathbf{6}$ & $\mathrm{Q} 6$ & $\mathrm{Q} 8$ & $\mathrm{Q} 6$ & $\mathrm{Q} 4$ & $\mathrm{Q} 13$ & $\mathrm{Q} 7$ & $\mathrm{Q} 7$ & $\mathrm{Q} 6$ \\
$\mathbf{7}$ & $\mathrm{Q} 7$ & $\mathrm{Q} 6$ & $\mathrm{Q} 13$ & $\mathrm{Q} 6$ & $\mathrm{Q} 12$ & $\mathrm{Q} 10$ & $\mathrm{Q} 8$ & $\mathrm{Q} 10$ \\
$\mathbf{8}$ & $\mathrm{Q} 8$ & $\mathrm{Q} 10$ & $\mathrm{Q} 3$ & $\mathrm{Q} 10$ & $\mathrm{Q} 15$ & $\mathrm{Q} 3$ & $\mathrm{Q} 10$ & $\mathrm{Q} 3$ \\
$\mathbf{9}$ & $\mathrm{Q} 9$ & $\mathrm{Q} 13$ & $\mathrm{Q} 10$ & $\mathrm{Q} 3$ & $\mathrm{Q} 10$ & $\mathrm{Q} 13$ & $\mathrm{Q} 13$ & $\mathrm{Q} 13$ \\
$\mathbf{1 0}$ & $\mathrm{Q} 10$ & $\mathrm{Q} 9$ & $\mathrm{Q} 14$ & $\mathrm{Q} 14$ & $\mathrm{Q} 17$ & $\mathrm{Q} 14$ & $\mathrm{Q} 11$ & $\mathrm{Q} 14$ \\
\hline
\end{tabular}

Fonte: Os autores.

Os dados completos na ordem final dos indicadores são apresentados na Tabela 3 (abaixo), que contém os dados apresentados na Figura 5.

Tabela 3 - Ordem final com prioridade de cada indicador para ser usado na avaliação de desempenho

\begin{tabular}{c|c|c|c|c|c|c|c|c}
\hline & $\mathbf{2 0 1 0}$ & $\mathbf{2 0 1 1}$ & $\mathbf{2 0 1 2}$ & $\mathbf{2 0 1 3}$ & $\mathbf{2 0 1 4}$ & $\mathbf{2 0 1 5}$ & $\mathbf{2 0 1 6}$ & $\mathbf{2 0 1 7}$ \\
\hline Q1 & 100,0 & 99,7 & 99,7 & 99,4 & 55,3 & 97,9 & 100,0 & 98,43 \\
Q2 & 97,50 & 100,0 & 100,0 & 100,0 & 62,5 & 100,0 & 99,8 & 100,0 \\
Q3 & 95,00 & 93,1 & 97,0 & 96,1 & 59,4 & 94,6 & 96,8 & 95,55 \\
Q4 & 92,50 & 98,5 & 97,5 & 98,0 & 67,0 & 99,6 & 96,9 & 98,89 \\
Q5 & 90,00 & 59,4 & 51,8 & 48,2 & 88,3 & 73,5 & 66,2 & 69,54 \\
Q6 & 87,50 & 92,3 & 97,4 & 97,3 & 71,9 & 96,8 & 95,1 & 97,23 \\
Q7 & 85,00 & 93,1 & 99,3 & 99,6 & 70,7 & 96,2 & 94,8 & 97,33 \\
Q8 & 82,50 & 92,6 & 97,6 & 98,3 & 77,3 & 97,7 & 93,6 & 97,86 \\
Q9 & 80,00 & 85,2 & 85,1 & 85,5 & 100,0 & 77,7 & 67,5 & 81,69 \\
Q10 & 77,50 & 86,5 & 96,6 & 96,8 & 81,9 & 94,9 & 91,3 & 95,84 \\
Q11 & 75,00 & 79,4 & 93,3 & 92,6 & 78,8 & 89,3 & 88,2 & 91,19 \\
Q12 & 72,50 & 79,3 & 92,1 & 91,8 & 85,5 & 91,1 & 87,2 & 92,03 \\
Q13 & 70,00 & 86,0 & 97,0 & 98,0 & 85,6 & 94,1 & 88,6 & 95,32 \\
Q14 & 67,50 & 81,6 & 93,6 & 94,3 & 91,4 & 93,2 & 86,5 & 93,83 \\
Q15 & 65,00 & 79,3 & 72,0 & 74,2 & 83,3 & 88,5 & 74,0 & 83,29 \\
Q16 & 62,50 & 74,0 & 89,5 & 89,7 & 92,5 & 88,3 & 82,3 & 89,41 \\
Q17 & 60,00 & 79,1 & 75,1 & 77,7 & 81,8 & 86,6 & 73,1 & 82,91 \\
Q18 & 57,50 & 72,9 & 86,6 & 87,4 & 86,7 & 84,4 & 78,0 & 85,6 \\
Q19 & 55,00 & 71,7 & 76,7 & 78,3 & 76,9 & 79,6 & 71,0 & 78,84 \\
Q20 & 52,50 & 75,0 & 85,9 & 87,8 & 81,7 & 82,6 & 74,9 & 83,75 \\
Q21 & 50,00 & 70,1 & 79,1 & 80,9 & 72,6 & 76,0 & 69,4 & 77,05 \\
Q22 & 47,50 & 68,5 & 81,6 & 83,2 & 75,1 & 75,5 & 69,7 & 77,56 \\
Q23 & 45,00 & 66,9 & 81,3 & 82,9 & 68,1 & 71,5 & 67,7 & 74,67 \\
Q24 & 42,50 & 65,3 & 78,9 & 80,7 & 69,2 & 70,5 & 65,5 & 73,18 \\
Q25 & 40,00 & 63,8 & 90,6 & 91,5 & 65,7 & 67,7 & 69,6 & 75,15 \\
Q26 & 37,50 & 62,2 & 84,4 & 85,8 & 65,7 & 66,3 & 65,5 & 72,12 \\
Q27 & 35,00 & 60,6 & 78,2 & 80,1 & 57,0 & 61,7 & 60,4 & 66,79 \\
Q28 & 32,50 & 59,0 & 72,0 & 74,4 & 57,0 & 60,2 & 56,3 & 63,76 \\
Q29 & 30,00 & 57,4 & 65,9 & 68,7 & 48,2 & 55,7 & 51,2 & 58,44 \\
Q30 & 27,50 & 55,8 & 59,7 & 63,0 & 48,3 & 54,2 & 47,1 & 55,41 \\
\hline
\end{tabular}

Fonte: Os autores.

Saindo agora da apresentação matemática do método, será apresentado abaixo um resumo das 
qualidades e defeitos do método.

\subsection{Resumo dos Pontos Positivos e Negativos para a Prática da Gestão}

Começando pelas dificuldades da proposta, como estamos falando de um algoritmo, é recomendado o desenvolvimento de uma plataforma WEB para a realização da pesquisa com banco de dados online para guardar os dados e fazer os cálculos, ou seja, é mais complicado do que meramente se criar uma tabela, no papel, com uma pontuação fixa, ou escala Likert, para ser distribuída entre as partes interessadas (stakeholders) e pedir seu preenchimento, para, na sequência, alguém juntar todos estes dados (papéis) e calcular a média.

Entretanto, os pontos positivos são vários. De forma genérica, tem-se que:

- A estrutura adaptativa faz com que os indicadores de desempenho mudem conforme a suave mudança entre as gerações da organização ou rapidamente após alguma mudança tecnológica. Além disso, por ter a capacidade de ser uma avaliação $360^{\circ}$ e usando-se da intersecção matemática entre os dados, diminui-se a possibilidade de indicadores mal intencionados serem usados. Estes podem mesmo ser zerados em casos de indicadores criados propositalmente para ajudar um grupo específico, quebrando naturalmente o início de uma avaliação corrupta;

- a publicização dos indicadores gera clareza na forma como será feita a avaliação, assim reduzindo entendimentos de "perseguição" ou de mal entendimento sobre as ações;

- o sistema contempla as indicações feitas por Lucena (1995), Zedeck e Cascio (1982), entre outros contidos na introdução deste artigo;

- ele é coparticipativo, e com isto, minimiza a possibilidade de as pessoas não aceitarem os indicadores selecionados, aumentando, dessa forma, a possibilidade de sucesso e gerando uma arquitetura de melhoria contínua quando realizado anualmente (ou sazonalmente).

- Especialmente para o serviço público: este sistema de seleção de indicadores quando agrupado a um sistema de avaliação de desempenho on-line faz com que a gestão consiga dados históricos das atividades realizadas como um dashboard ou um sistema de supervisão industrial, um SCADA (Supervisory Control And Data Acquisition) e com isso os cursos de capacitação não precisam mais ser feitos por uma pesquisa do que os servidores gostariam de fazer, mas sim com o que eles mais precisam para dar o melhor retorno nas suas atividades. Com todos estes itens considerados, existe uma forte efetividade nos recursos públicos investidos na gestão de pessoas;

- Como o sistema é adaptativo, ele é robusto a pequenos erros de modelagem inicial, ao final convergindo para o conjunto de indicadores coparticipativos mais adequados para cada instituição, para cada nível e para cada área.

\section{Considerações Finais}

Os sistemas de avaliação de desempenho são complexos e possuem uma tendência natural ao insucesso quando tratados de forma simplista. Para o controle do sistema complexo chamado de avaliação de desempenho, optou-se, neste artigo, pelo uso das ferramentas existentes na Engenharia de Controle de Sistemas, uma vez que esta área do conhecimento costuma trabalhar com um grande número de variáveis, restrições de todos os tipos, correlações complexas, mas com a obrigatoriedade de apresentar resultados concisos e que possuam repetibilidade.

Nos dados simulados, não foram visualizados erros ou discrepâncias para os oito anos propostos. Mesmo quando foi propositalmente inserido um erro (distúrbio), o sistema voltou a se estabilizar, o que demonstra sua eficácia e robustez.

0 algoritmo proposto demonstrou que esta metodologia pode ser amplamente usada para ranquear e selecionar, com ordem de prioridade, as melhores práticas (ou indicadores) para serem usadas como critérios para a avaliação de desempenho em instituições públicas, coparticipativas e afins, melhorando, dessa forma, os resultados organizacionais com a mesma precisão de um sistema eletromecânico, entretanto, sempre se pautando nas especificidades do grupo de forma limpa e transparente.

Com base nos resultados positivos da simulação, indica-se como próxima etapa desta pesquisa, a construção de uma plataforma on-line (WEB) que permitirá a seleção de parâmetros e a avaliação dos usuários. Aplicando-se a metodologia a instituições, áreas e níveis diferentes será possível verificar os limites da eficiência desta arquitetura de avaliação adaptativa. 
Em trabalhos futuros sobre avaliação de desempenho usando ferramentas da engenharia, recomenda-se uma proposta que se utilize de uma arquitetura de avaliação de atividades intelectuais, item fundamental para a área de ensino universitário e pesquisa científica respeitando as diferenças entre nível, área e idade das instituições (itens indissociáveis).

Entende-se que as técnicas de controle de sistemas quando acopladas a bases de dados e comunicação on-line podem gerar uma plataforma WEB de melhoria contínua da instituição, com baixos custos de investimento a longo prazo, ou seja, existem indícios de que esta interdisciplinaridade pode contribuir para pesquisas e produtos futuros uma vez que, como apresentado na revisão da literatura, muitos autores indicam teoricamente como deve ser a avaliação, mas poucos, ou nenhum, conseguiu apresentar ferramentas e métodos que contemplassem com avaliar, controlar, motivar, aprender e melhorar.

\section{Referências}

Aguiar, G. F. (2003). Avaliação da eficiência técnica de unidades básicas de saúde em Curitiba-PR utilizando DEA. (Dissertação de mestrado). Universidade Federal do Paraná, Setor de Tecnologia, Programa de Pós-Graduação em Métodos Numéricos em Engenharia. p.121.

Aström, K. J., \& Wittenmark, B. (2008). Adaptive control (2ª ed.). Mineola, NY: Dover Publications. Behn, R. D. (2003). Why measure performance? Different purposes require different measures. Public Administrations Review, Vol. 63 No. 5, pp. 586-606.

Bourne, M., Mills, J., Wilcox, M., Neely, A., \& Platts, K. (2000). Designing, implementing and updating performance measurements systems. International Journal of Operations \& Production Management. V. 20, N. 7. pp. 754-771.

Deming, W. E. (1989). O método Deming de Administração (5ª ed.). São Paulo: Saraiva.

Edwards, M. R., \& Ewen, A. J. (1996). 360º Feedback: The powerful new model for employee assessment \& performance improvement. New York: Amacon.

Galindo, R. W. (2017, 23 de agosto). Por que o serviço público não funciona. In: GAZETA DO POVO. Retirado de http://www.gazetadopovo.com.br/vida-publica/por-que-o-servico-publico-naofunciona-9684xpuwd5lvz841xc68w7ham.

Hesketh, B., \& Neal, A. (1999). Technology and performance. In D.R. Ilgen \& D.P. Pulakos (Eds.), The changing nature of performance: Implications for staffing, motivation and development (pp. 21-55). San Francisco: Jossey-Bass.

Kaplan, R. S., \& Norton, D. P. (1997). A estratégia em ação: Balanced Scorecard (13ạ ed.). Rio de Janeiro: Campus.

London, M., \& Mone, E. M. (1999). Continuous Learning. In D. R. Ilgen \& D. P. Pulakos (Eds.), The changing nature of performance: Implications for staffing, motivation and development (pp. 119153). San Francisco: Jossey-Bass.

Lucena, M. D. da S. (1995). Avaliação de desempenho. São Paulo: Atlas.

Machado, N., Holanda, V. B. De, Ribeiro Filho, J. F., Lopes, J. L., \& Pederneiras, M. (2012). Gestão baseada em resultado no setor público: Uma Abordagem Didática para Implementação em Prefeituras, Câmaras Municipais, Autarquias, Fundações e Unidades Organizacionais. São Paulo: Atlas.

Marr, B. (2012). Key Performance Indicators (KPI): The 75 measures every manager needs to know (Financial Times Series). FT Press. p. 375.

Martin, J., Elg, M., Wallo, A.\& Kock, H. (2018). Four facets of learning in performance measurement. International Journal of Productivity and Performance Management, Vol. 67 No. 9, pp. 1608-1624. https://doi.org/10.1108/IJPPM-11-2017-0320

Megginson, L. C., Mosley, D. C., \& Pietri, H. (1998). Administração: conceitos e aplicações (4⿳亠丷a ed.). São Paulo: Harbra.

Missel, S. (2012). Feedback Corporativo: como saber se está indo bem. São Paulo: Saraiva.

Murphy, P. R., \& Jackson, S. E. (1999). Managing work role performance: Challenges for twenty-firstcentury organizations and their employees. In D. R. Ilgen \& D. P. Pulakos (Eds.), The changing nature of performance: Implications for staffing, motivation and development (pp. 325-365). San Francisco: Jossey-Bass.

Oliveira-Castro, G. A. (1994). Avaliação de desempenho em psicologia: questões conceituais e metodológicas. Psicologia: teoria e pesquisa, v. 10, n. 3.

Oliveira-Castro, G. A., Lima, G. B. C., \& Veiga, M. R. M. (1996). Implantação de um sistema de avaliação de 
desempenho: Métodos e estratégias. São Paulo. Revista de Administração, v. 31, n.3.

Oliveira, A. A. de. (2016). Software Mensures: Mensurador de atividades intelectuais. Registro do processo: BR 512016 001521-7. Curitiba. Instituto Nacional de Propriedade intelectual - INPI.

Oliveira, A. A. de. (2017) Pesquisa sobre indicadores para a geração de feedback automático do trabalho docente. Relatório de pesquisa de junho de 2016 a junho de 2017. Curitiba: Instituto Federal do Paraná (IFPR).

Oliveira, A. A. de., Deschamps, F., \& Lima, E. P. de. (2016). Adaptive Control Applied to the Performance Management Function of Organizations. In: ISPE International Conference on Transdisciplinary Engineering, Curitiba. Advances in Transdisciplinary Engineering, 2016. v. 4. p. 768-777. Retirado de http://ebooks.iospress.nl/volumearticle/45462.

Parmenter, D. (2010). Key Performance Indicators (KPI): Developing, Implementing, and Using Winning KPIs ( $2^{\circ}$ ed). Ed. Wiley.

Parmenter, D. (2012). Key Performance Indicators for Government and Non Profit Agencies: Implementing Winning KPIs. Ed. Wiley. p. 336.

Rao, S. S. (2009). Engineering optimization: theory and practice. 4th ed. Hoboken, N. J.: John Wiley. xix, p. 813.

Ribeiro, G. (2017). Avaliação de desempenho: instrumento de dominação ou de gestão organizacional. São Carlos: Scienza.

Rosen, M., Bedwell, W. L., Wildman, J. L., Fritzsche, B. A., Salas, E., \& Burke, C. S. (2011). Managing adaptive performance in teams: Guiding principles and behavioral markers for measurement. Human Resource Management Review, 21(2), 107-122. https://doi.org/10.1016/j.hrmr.2010.09.003

Stoffel, I. (1997). Administração do desempenho: metodologia gerencial de excelência. Florianópolis: Perspectiva.

Tao, G. (2014). Multivariable adaptive control: A survey, Automatica. V. 50, Issue 11, November. p. 2737-2764.

The KPI Institute. (2013). The KPI Compendium: 20,000 Key Performance Indicators used in practice Paperback. Create Space Independent Publishing. p. 300.

Vasconcellos, E., \& Krugliankas, I. (1991). Avaliação de desempenho em instituições de pesquisa. São Paulo: Revista de Administração, v. 26, n. 4.

Zedeck, S., \& Cascio, W. F. (1982). Performance appraisal decisions as a function of rates training and purpose of the appraisal. Journal of applied psychology, v. 67, n. 6. 